\title{
COMMON FUNGUS INFECTIONS OF THE SKIN
}

\author{
By I. R. MARRE, M.R.C.S., L.R.C.P.
}

Skin Physician to the Acton, Evelina and Metropolitan Hospitals.

The common superficial mycoses of the skin are caused by a number of different organisms, and I propose to give a short general description of the types of fungi usually responsible for these infections, before proceeding to the question of diagnosis and treatment.

There are three groups to be considered :-

I. Ringworm fungi.

2. Monilia.

3. Other fungi.

1. Ringworm fungi. This is a very large class, but we need refer only to the following types:-

(a) Microspora:

(b) Trichophyta.

(c) Achoria.

(d) Epidermophyta.

The microspora have small spores in mosaic arrangement, and are found in profusion on the hair surface. They are responsible for most of the scalp ringworm in this country, and are either conveyed by human infection $(M . a u$ douini-the largest group), or from animals ( $M$. lanosum). Infection with $M$. audouini tends to die out at puberty, and only occasionally attacks the glabrous skin. Infection with $M$. lanosum generally shows more reaction, and on the glabrous skin cause well-marked rings.

The trichophyta are large-spored ringworm, and are divided into two groups, the endothrix and the ectothrix. The endothrix group are of human origin and grow in large-spored chains within the hair, but the ectothrix group, of animal origin, grow on the hair surface and within the hair as well. The trichophyta also attack the glabrous skin, beard and nails, and produce a vigorous reaction, frequently going on to pustulation.

The Achoria are responsible for the production of favus, characterized by the occurrence of yellowish, cup-shaped scutula. It commonly affects the scalp, but may affect the hair, glabrous skin or nails. The usual organism is A. Schönleini, but the group is nowadays usually placed in the endothrix trichophyta.

The epidermophyta never affect the hair. They are commonly responsible (especially the $E$. inguinale) for infection of the crural region (Tinea cruris or Dhobie Itch), between the toes and in the nails.

2. Monilia. These are a group of yeast-like? fungi, of which the usual one is $M$. albicans. Unlike the ringworm fungi, the manifestations of which are usually fairly localized, monilia are capable of serious generalized and systemic infections. Monilia have a predilection for folds and flexures, and the infection is often characterized by moist whitish patches.

3. Other fungi. We need only concern ourselves here with two :-

(a) Microsporon furfur.

(b) Microsporon minutissimum.

(a) Microsporon furfur is the cause of Pityriasis Versicolor (Tinea Versicolor). It is a yeast-like fungus.

(b) Microsporon minutissimum. This exceedingly minute organism, only seen with $1 / 12$ in. oil immersion lens, is the cause of erythrasma, and is really of the actinomyces group.

In discussing the superficial fungus infections, it is most convenient to divide the body into regions, and to describe the common in- 
fections in each area. We shall consider the following regions :-

I. The scalp.

2. The beard.

3. The glabrous skin.

4. The flexures.

5. The feet.

6. The nails.

\section{The Scalp}

In this country the most common type is the small spored infection with $M$. audouini, and it does not, unfortunately, respond to local treatment. It usually starts as a small whitish-grey scaly patch, which spreads and forms a mildly inflamed circular lesion, covered with short, broken, twisted and bent stumps about $\frac{1}{6}$ in. long. There may be several such small patches, and after a time large areas are affected by coalescence. If untreated the condition persists until puberty, when it tends to die out. Its appearance may be altered by scratching and infection.

Endothrix trichophyton is a common cause of " black-dot" ringworm, the stumps breaking off at scalp level. They should be elevated with a comedo-expressor for removal and examination.

Ectothrix trichophyton is characterized by a violent inflammatory reaction, usually with the development of a Kerion. This is a welldefined boggy swelling, with a red inflamed surface, from which small follicular abscesses ooze beads of pus. There is a tendency to selfcure here, as the infected hairs become loose and tend to fall out, so that eventually a smooth, bald patch is left. If there has been scarring the baldness may be permanent, but usually hair grows again.

Favus starts as a scaly spot with minute yellow spots at the hair follicles. These spread and become yellow, cup-shaped crusts or scutula, with concave side upwards. The hair is brittle and lustreless, but does not show much tendency to break into stumps. The disease spreads slowly if untreated, with an end result of permanently bald scarred patches.

Diagnosis. This should be confirmed in. every case by examination of affected hairs in $\frac{0}{0}$ 30 per cent. potassium hydroxide solution, when spores or mycelial threads will be seen under $\frac{1}{6}$ in. objective. Gentle warming after immersion of the hair in the $\mathrm{KOH}$ solution facilitates examination.

Infection with Microsporon shows fluorescence under Woods' light (filtered ultra-violet light), but other types of scalp ringworm are difficult to recognize by this means.

Other conditions may resemble tinea superficially, but reveal no fungus on examination. Alopecia areata is a non-scaly completely bald patch, with upright " exclamation mark " hairs at the periphery. Seborrhoeic dermatitis has. yellow greasy scales with no patchy hair loss. Psoriasis has a red inflamed base, covered with typical silvery scales, and no loss of hair. Pediculosis capitis is a common cause of scalp sepsis in children.

Treatment. Local measures should be tried with $M$. lanosum and ectothrix trichophyton (Kerion) infection, as these are often successful, and avoid epilation. The patches may be $\vec{\bullet}$ painted twice daily with Pigmentum Castellar (Fuchsin paint), or rubbed with Whitfield ointment (Ung. Ac. Benzoic. B.P.C.). Tho formation of a Kerion may be encouraged by rubbing in a 33 per cent. soldium chloride ointment.

With the other and more resistant types of scalp ringworm, epilation must be carried out, and three methods may be used.

(a) Manual epilation. This is useful for small single patches, and epilation with forceps is carried out every three or four $\underline{3}$. days. The rest of the scalp is kept covered with an antiseptic ointment, such as Ung. Hydrarg. Ammon.

(b) $X$-ray epilation. Is most successful $\frac{?}{0}$ and efficient in capable hands. After the epilating dose a mild mercury ointment is o applied to the scalp, and frequent shampoos $\tilde{O}$ to assist hair fall. X-ray is difficult to use in $\underset{\mathrm{N}}{\mathbb{N}}$ children under the age of 4 or 5 , as they do not keep still.

(c) Thallium acetate epilation. The method $\stackrel{\infty}{\stackrel{\oplus}{\rightarrow}}$ consists in the administration by mouth of 80 mgm: of thallium acetate per kilogram of $\frac{\vec{D}}{\mathbb{D}}$ 
body weight. The drug is dangerous, as a mistake in the dose may lead to the death of the patient, and it is used only for children as it is highly toxic to adults.

\section{The Beard}

This is an uncommon infection, and usually there are other foci on the body. There are two types :-

(a) Kerion or inflammatory type due to animal ectothrix trichophyton.

(b) Dry scaly type, usually an endothrix of human origin, which may spread peripherally, or form a follicular sycosis.

Diagnosis. Rests on demonstration of fungus. The condition must be differentiated from coccal sycosis barbae, in which there are no stumps with fungus, and also from granulomatous eruptions due to iodides and bromides, to syphilis and to actinomycosis.

Treatment. Initial sepsis and pustulation may be dealt with by hypertonic saline compresses, or boric acid lotion. When this has subsided, manual epilation may be undertaken, together with a twice daily application of a fungicide such as Whitfield's ointment or Castellani's paint. Epilation by X-ray has been used in this area but is usually unnecessary.

\section{The Glabrous Skin}

The appearance of the infection here varies considerably with the infecting fungus. There is a macular scaly eruption which may be due to $M$. audouini or an endothrix trichophyton. The classical eruption of circinate ringed lesions is caused by $\boldsymbol{M}$. lanosum. This is a ringed scaly lesion, with peripheral vesiculation, which increases in diameter, clearing in the centre, and often joining up with other patches to form gyrate figures. An eczematous type is formed by the ectothrix trichophyton, at first vesicular and later pustular. A solid plaque type resembling psoriasis is also found on the body, as well as a granulomatous type, both due to trichophyta. Favus causes a crusted type of lesion, with the tendency to formation of yellowish scutula.

Pityriasis versicolor (due to $M$. furfur) is a cafè-au-lait coloured macular eruption, with a fine branny scaling, affecting usually the trunk, axillae and neck. It begins as irregular fawnish macules, which coalesce to form diffuse sheets, and are separated by paler areas of normal skin. It is said to occur particularly in cachectic patients who perspire freely, and therefore is common in tuberculosis.

Diagnosis. This is simple in the circinate variety of ringworm, but may be much more difficult in the eczematous or plaque types. Demonstration of the fungus in ro per cent. potassium hydroxide is certain. Pityriasis versicolor shows abundant fungus in scrapings of the scales.

Pityriasis rosea can be differentiated by the herald patch, together with the sudden secondary eruption on the body and upper parts of the arms and legs, and no fungus can be demonstrated.

Contact dermatitis is differentiated by the history and lack of circinate outline. The plaque type must be recognized from psoriasis with its silvery scales and bleeding points, and from neurodermatitis where there is no evidence of fungus disease elsewhere.

Final and certain diagnosis lies in recog nition of the fungus microscopically.

Treatment. Tinea of the glabrous skin usually responds well to treatment. Castellani's pigment painted on twice daily for a week suffices, or Whitfield's ointment may be used. Tincture of iodine (weak tincture) may be used in the same way.

Pityriasis versicolor responds to an ointment of 2 per cent. sulphur and salicylic acid, together with frequent bathing and changing of underclothes. Treatment must be persisted with for at least two weeks after apparent cure, as the condition is resistant.

After cure a course of general ultra violet light is useful in preventing a recurrence.

\section{The Flexures}

Here we are concerned mainly with the groins, internatal cleft, submammary region in the female, and the axillae.

Tinea cruris, or Dhobie Itch, is a common type of ringworm, usually confined to the upper and inner parts of the thighs. It may 
spread to the genitals and the internatal cleft, and is also found in the submammary and axillary areas. If specifically sought, there is nearly always a primary focus between the toes, and this must be treated simultaneously. The condition is usually due to Epidermophyton Inguinale, and is a well-marginated scaly rash spreading peripherally from the flexures.

Erythrasma is caused by a tiny fungus ( $M$. minutissimum), and gives rise to well-defined reddish-yellow or reddish-brown patches especially in the crural regions and the axillae. There is a faintly scaly surface, and the fungus is found in the scales (with $1 / 12$ in. oil immersion lens).

Monilia grow best in warm, moist places, and, therefore, favour the intertriginous areas, especially in fat people, and in diabetics. The eruption starts as small macules or vesicles, which coalesce to form irregular red patches, bounded by an edge of detached skin. Macerated patches of whitish epithelium may be found in association with the condition, especially in the internatal cleft. Moniliasis has special names $(a)$ in the webs of the fingers, and $(b)$ in the angles of the mouth.

(a) Erosio interdigitalis blastomycetica. A tender infection of the web of the fingers, with a moist, red base, surrounded by detached skin.

(b) Perlèche. An intertrigo affecting the angle of the mouth. In infants infection of the buccal mucosa with monilia is known as thrush, and may affect the whole gastrointestinal tract; in adults a glossitis and stomatitis has been described. Monilia is a common cause of pruritus vulvae and pruritus ani.

A chronic form of paronychia is due to infection of the nail fold with monilia, with red and moderately tender swelling of the paronychial tissues. "This condition is found particularly among people who keep their hands in water, such as housewives and barmaids, and it is probable that continual maceration of the skin is a factor.

Diagnosis. Tinea cruris and erythrasma may be confused, but the former has a festooned marginated border, tends to central clearing and spreads more rapidly. Microscopic examina- tion will confirm the diagnosis. The picture in moniliasis is usually distinctive with its red centre and thin detached edge, and the yeastlike fungus will be found in the free edge of the lesion. Acute coccal paronychia is much more painful and acute than the chronic monilial type.

Treatment. The epidermophyton (tinea cruris) usually responds well to simple fungicides such as Whitfield's ointment or Pigmentum Castellani.

Erythrasma responds well to the twice daily inunction of a 2 per cent. sulphur and salicylic ointment.

Monilia is best treated with a I per cent. aqueous solution of gentian violet, or for small areas a I per cent. solution in 25 per cent. spirit. Monilial paronychia may be treated in the same way with a 2 per cent. solution of gentian violet in 25 per cent. spirit introduced into the nail fold twice daily with an orange stick.

\section{The Feet}

Here, too, different types of lesions are pro duced by different organisms. $\boldsymbol{E}$. inguinale produces scaly patches between the toes and on the soles, with the usual sharply marginated border. Trichophyta produce vesicular eruptions on the soles and sides of the feet, whereas monilia produce macerated cracks between the toes. The feet are often secondarily infected, and this must be attended to first. Hyperkeratotic patches on the feet may be impregnated with fungus and must be treated.

Diagnosis. If all the webs of the toes are affected, and the epithelium is macerated, the infection is monilia, whereas if the fourth space shows a scaly eruption, it is likely to be $E$. inguinale. Contact dermatitis from shoes and from medicaments is common, and may be distinguished from fungus by the site, history and lack of microscopical evidence. Psoriasiform fungus infections are seen and are differentiated from true psoriasis by the lack of psoriasis elsewhere, by more intense itching and by demonstration of the fungus.

Treatment. Infection usually responds well to footbaths twice daily with $\mathrm{I}: 5000$ potassium permanganate. The affected areas are allowed 
to rest in the lukewarm bath for 20 minutes and to dry in the air. Later both epidermophyta and trichophyta respond well to Whitfield's ointment or Castellani's paint, and the monilia to I per cent. gentian violet. The greatest care to prevent re-infection must be taken. Cotton socks should be worn beneath ordinary socks or stockings, and boiled and changed daily. Hyperkeratotic areas may be treated with 6 per cent. salicylic acid ointment, until they are flat, and later with the usual fungicides.

Footwear may be sterilized by shutting in an air-tight box with formaldehyde, so that the vapour is allowed to act for four or five days.

\section{The Nails}

Ringworm of the nails, or onychomycosis, may begin independently, but often occurs with fungus infection elsewhere on the body. Infection usually takes place at the free edge of the nail, when the nail bed is attacked, and a dirty grey scaly thickening raises the nail from its bed. It spreads down the nail and grows up into the nail plate, which becomes discoloured and thickened, and finally crumbles. Infection may, however, pass under the lateral border of the nail, first attacking one side with ridging of the nail, but gradually passing across and affecting the whole nail. The infection is due to trichophyta or epidermophyta.

Diagnosis. Psoriasis of the nails may resemble fungus closely, but is not so irregular, and no fungus can be demonstrated. Syphilis of the nails is usually associated with signs elsewhere.

Treatment. Fungus infection of the nails is difficult to cure and demands unremitting attention on the part of the patient. In early infection, with only part of the nail affected, this part should be scraped away with a piece of broken glass or sharp knife until there is no obvious infection. This should be done daily, and Whitfield's ointment is applied under strapping each night. Persistence with this treatment, for months if necessary, will result in a cure.

If much of the nail is affected, surgical evulsion should be performed, and the thickened nail bed scraped. After a few days, continuous application of Whitfield's ointment should be insisted on until the nail is fully grown.

Ringworm fungi of the body, and particularly of the feet, are capable of sensitizing a patient, with the production of an allergic or sensitization eruption, which is itself free from fungus. A lichenified dermatophytide (or mycide) has been described on the trunk due to tinea capitis, but more characteristic in ringworm infection is the 'pompholyx' type of eruption on the hands. This consists of tiny? vesicles along the sides of the hand, on the palms and between the fingers, and these vesicles contain no fungus. Treatment of the primary focus results in the spontaneous clearing up of the secondary sensitization eruption.

\section{CORRECTION}

Vol. XXIII, No. 257. March 1947, page 165.

\section{SEBORRHOEIC DERMATITIS :-}

Treatment.

The first formula given should be corrected to read :-

$$
\begin{aligned}
& \text { R Acidi Salicylici .......... gr. } 20 \\
& \text { Hydrargyri Perchloridi ..... gr. } \frac{1}{2} \\
& \text { Liq. Carbonis deterg. .... m. I5 } \\
& \text { Olei ricini ............. m. Io } \\
& \text { Spirit vini rect. or spirit meth. } \\
& \text { indust. } \ldots \ldots \ldots \ldots \ldots \text { ad. } \overline{3} \mathrm{I}
\end{aligned}
$$

